\title{
一种响应速率匹配的双反应探针用于荧光识别硫化氢
}

\author{
解畅马趁贾旭张学琪 \\ 魏超* 张平竹李小六* \\ (河北大学化学与环境科学学院 河北省化学生物学重点实验室 \\ 药物化学与分子诊断教育部重点实验室 河北保定 071002)
}

\begin{abstract}
摘要 以 6-氟-7-氨基香豆素为苂光基团, 氟代叠氮和 7-硝基苯并氟咱(NBD)-哌嗪为 $\mathrm{H}_{2} \mathrm{~S}$ 反应基团和苂光淬灭基团, 合 成一种双反应 $\mathrm{H}_{2} \mathrm{~S}$ 荧光探针. 探针对 $\mathrm{H}_{2} \mathrm{~S}$ 的识别性质研究表明, 探针的两个反应基团与 $\mathrm{H}_{2} \mathrm{~S}$ 响应速率匹配, 探针对 $\mathrm{H}_{2} \mathrm{~S}$ 具有高选择性和灵敏性, 其苂光增强约 3600 倍, 检测极限为 $4.0 \times 10^{-8} \mathrm{~mol} / \mathrm{L}$. 酶活性测试表明, 探针可用于胱硫醚 $\beta$ 合成酶(CBS)酶活性检测和抑制剂笁选. 细胞成像实验表明, 探针可用于细胞内 $\mathrm{H}_{2} \mathrm{~S}$ 的成像研究.

关键词＼cjkstart硫化氢; 响应速率; 双反应; 荧光探针
\end{abstract}

\section{A Response Rate Matching Dual-Reactable Probe for Fluorescent Recognition of Hydrogen Sulfide}

\author{
Xie, Chang Ma, Chen Jia, Xu Zhang, Xueqi Wei, Chao* \\ Zhang, Pingzhu Li, Xiaoliu*
}

(Key Laboratory of Chemical Biology of Hebei Province, Key Laboratory of Medicinal Chemistry and Molecular Diagnosis of Ministry of Education, College of Chemistry and Environmental Science, Hebei University, Baoding, Hebei 071002)

\begin{abstract}
A dual-reactable $\mathrm{H}_{2} \mathrm{~S}$ fluorescent probe was designed and synthesized by employing ortho-fluoro-substituted coumarin azide and 7-nitrobenzofurazan-piperazine as the $\mathrm{H}_{2} \mathrm{~S}$ reactive groups and the fluorescence quenching groups. The recognition behaviors of the probe to $\mathrm{H}_{2} \mathrm{~S}$ were investigated and the results showed that the probe exhibited high selectivity and sensitivity. The fluorescence off-on enhancement was $c a$. 3600 -fold, and the detection limit was $4.0 \times 10^{-8} \mathrm{~mol} / \mathrm{L}$. The results of enzyme activity test indicated that the probe could be used for cystathionine $\beta$-synthase (CBS) activity detection and inhibitor screening. Furthermore, the probe was successfully applied for the imaging of $\mathrm{H}_{2} \mathrm{~S}$ in living cells.

Keywords hydrogen sulfide; response rate; dual-reactable; fluorescent probe
\end{abstract}

硫化氢 $\left(\mathrm{H}_{2} \mathrm{~S}\right)$ 具有独特的臭鸡蛋气味, 是一种有毒 环境污染物. 近年来研究发现, $\mathrm{H}_{2} \mathrm{~S}$ 是继一氧化碳 $(\mathrm{CO})$ 和一氧化氮(NO)之后的第三种内源性气体信号递质分 子 $^{[1]}$. 在哺乳动物不同组织和器官中, 内源性 $\mathrm{H}_{2} \mathrm{~S}$ 可由 胱硫醚 $\beta$ 合成酶 $(\mathrm{CBS}) 、$ 胱硫醚 $\gamma$ 裂解酶、3-颈基丙酮酸 硫转移酶(3-MPST)/半胱氨酸氨基转移酶(CAT) 以及氨 基酸氧化酶(DAO)催化手性半胱氨酸生成 ${ }^{[2]}$. 研究表明, 体内 $\mathrm{H}_{2} \mathrm{~S}$ 浓度异常与许多疾病有关, 包括阿尔茨海默氏
症、唐氏综合症、糖尿病和肝硬化等 ${ }^{[3]}$. $\mathrm{H}_{2} \mathrm{~S}$ 被认为与多 种生理和病理过程有关 ${ }^{[4]}$, 其在体内的潜在分子机制尚 不明确 ${ }^{[5]}$. 因此, 开发能够有效检测生物体内 $\mathrm{H}_{2} \mathrm{~S}$ 的分 析方法具有重要的研究意义 ${ }^{[6]}$.

与传统的 $\mathrm{H}_{2} \mathrm{~S}$ 检测方法相比, 苂光探针技术具有选 择性好、灵敏度高等特点, 尤其是对生物系统无侵入性 损伤、适于细胞及活体成像, 因此被广泛用于检测内源 性生物活性分子 ${ }^{[7]}$. 基于硫化氢的还原性和亲核性，多

\footnotetext{
* Corresponding authors. E-mail: weichao@hbu.edu.cn; lixl@hbu.cn Received May 16, 2019; revised June 24, 2019; published online July 17, 2019.

Project supported by the National Natural Science Foundation of China (No. 21778013), the Natural Science Foundation of Hebei Province (No. B2018201234), the Colleges and Universities Science Technology Research Project of Hebei Province (No. QN2017015), the Science Technology Research and Development Guidance Programme Project of Baoding City (No. 16zg031) and the Open Fund of Laboratory in Hebei University (Nos. sy201833, KYZJX18144).

国家自然科学基金(No. 21778013)、河北省自然科学基金(No. B2018201234)、河北省高等学校科学技术研究(No. QN2017015)、保定市科学研究与发 展计划(No. 16zg031)和河北大学实验室开放(Nos. sy201833, KYZJX18144)资助项目.
} 
种反应类型的 $\mathrm{H}_{2} \mathrm{~S}$ 苂光探针被发展出来, 主要有芳香叠 氮/硝基还原型 ${ }^{[8]}$ 、亲核取代型 ${ }^{[9]}$ 、亲核加成/双亲核加成 型 ${ }^{[10]}$ 和形成 $\mathrm{CuS}$ 沉淀型 ${ }^{[11]}$ 等. 2013 年, 我们 ${ }^{[9 \mathrm{~d}]}$ 发现 7硝基苯并氟咱(NBD)-哌嗪结构可以选择性与 $\mathrm{H}_{2} \mathrm{~S}$ 发生 亲核取代(硫解)反应, 并构建了基于苂光共振能量转移 (FRET) 原理的 $\mathrm{H}_{2} \mathrm{~S}$ 苂光探针 1. 同时, 发现在叠氮基香 豆素结构中引入吸电子基团氟原子, 可以在保持探针选 择性的同时, 提高叠氮被 $\mathrm{H}_{2} \mathrm{~S}$ 还原的速率 $(2 \text { 和 } 3)^{[\mathrm{gg}]}$. 进 一步, 利用双淬灭效应(NBD 参与的 FRET 淬灭和叠氮 参与的 ICT 淬灭)增加苂光淬灭程度, 当探针和 $\mathrm{H}_{2} \mathrm{~S}$ 反应 后, 双淬灭效应同时消失, 苂光增强明显, 可显著提高 探针的灵敏性. 充分利用 $\mathrm{H}_{2} \mathrm{~S}$ 分子的强还原性和强亲核 取代性，降低单反应性分子的竞争性干扰，显著提高探 针的选择性. 基于此设计理念, 发展了 FRET-ICT 双反 应 $\mathrm{H}_{2} \mathrm{~S}$ 苂光探针 $\mathbf{4}^{[12]}$.<smiles>CCN(CC)c1ccc2cc(C(=O)N3CCN(c4ccc([N+](=O)[O-])c5nonc45)CC3)c(=O)oc2c1</smiles><smiles>[Y][X]c1cc2oc(=O)cc(CC(=O)N3CCNCC3)c2cc1[Y]#[Y]</smiles><smiles>[H][R17](=O)c1cc([N+](=O)[O-])c2nonc2c1N1CCN(C(=O)Cc2cc(=O)oc3cc(N)c(CC)cc23)CC1</smiles><smiles>Nc1cc2oc(=O)cc(CC(=O)N3CCN(c4c(CC(=O)O)cc([N+](=O)[O-])c5nonc45)CC3)c2cc1F</smiles>

图 1 已报道的 $\mathrm{H}_{2} \mathrm{~S}$ 苂光探针 $\mathbf{1} \sim \mathbf{4}$ 和本工作设计的苂光探针

\section{5}

Figure 1 Reported $\mathrm{H}_{2} \mathrm{~S}$ fluorescent probe $\mathbf{1} \sim \mathbf{4}$ and probe 5 in this work

双反应探针与 $\mathrm{H}_{2} \mathrm{~S}$ 的反应速率取决于反应活性较 低的基团, 而探针 2 的反应速率 $\left(k_{2}=6.95 \mathrm{~L}^{\circ} \cdot \mathrm{mol}^{-1} \cdot \mathrm{s}^{-1}\right)$ 明显低于探针 1 的反应速率 $\left(k_{2}=15.5 \mathrm{~L} \cdot \mathrm{mol}^{-1} \cdot \mathrm{s}^{-1}\right)$, 为 了提高双反应探针的反应速率，将邻位氟代叠氮基香豆 素 3(反应速率 $k_{2}=13.2 \mathrm{~L} \cdot \mathrm{mol}^{-1} \cdot \mathrm{s}^{-1}$ ) 与 $\mathrm{NBD}-\mathrm{Cl}$ 反应，经 一步反应制备了双反应菼光探针 5 (Eq. 1), 测试了探针
的选择性、灵敏性、与 $\mathrm{H}_{2} \mathrm{~S}$ 反应速率及光/热稳定性等 光谱性能, 初步探索了探针的实际应用能力 (CBS 酶活 性测试和细胞内 $\mathrm{H}_{2} \mathrm{~S}$ 荧光成像).<smiles>Nc1cc2oc(=O)cc(CC(=O)N3CCNCC3)c2cc1F</smiles>

\section{1 结果与讨论}

\section{1 识别性能}

在 $37{ }^{\circ} \mathrm{C}$ 向 $1.0 \times 10^{-6} \mathrm{~mol} / \mathrm{L}$ 探针 $\mathbf{1} \sim \mathbf{5}$ 的磷酸缓冲 盐(PBS)溶液 $\left(1.0 \times 10^{-2} \mathrm{~mol} / \mathrm{L}, \mathrm{pH} 7.4\right.$, 含 $10 \%$ 乙腈)中 加入 $2.5 \times 10^{-4} \mathrm{~mol} / \mathrm{L} \mathrm{H}_{2} \mathrm{~S}$, 测试了探针溶液随时间变化 的苂光发射光谱. 随着反应进行，探针 5 苂光强度明显 增强. 反应结束后, 苂光增强倍数高达 3600 倍(图 2a).

以
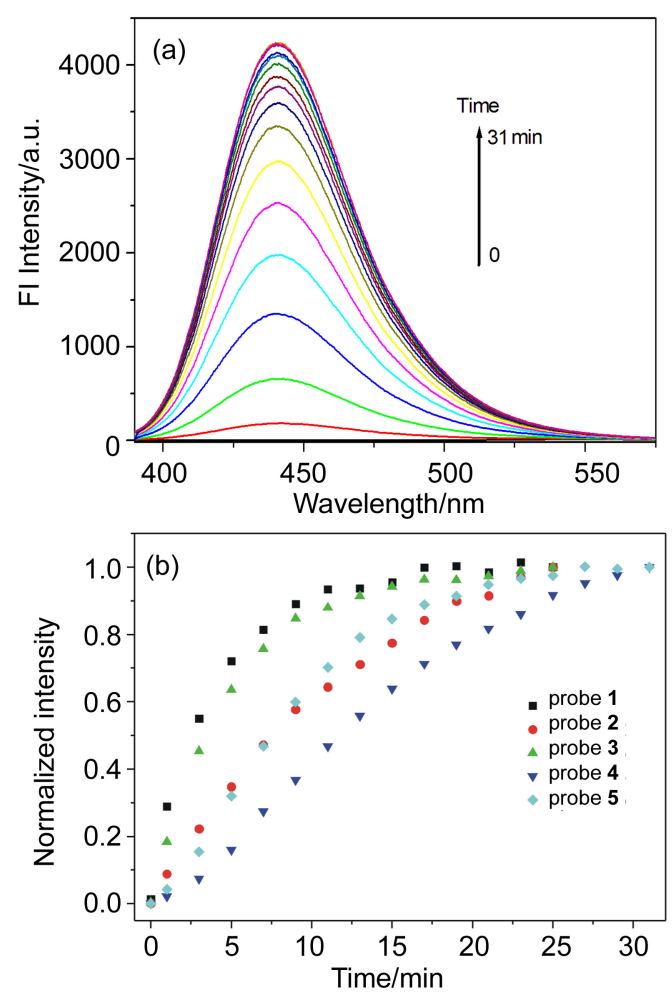

图 2 探针 $\mathbf{1} \sim \mathbf{5}\left(1.0 \times 10^{-6} \mathrm{~mol} / \mathrm{L}\right)$ 与 $\mathrm{H}_{2} \mathrm{~S}\left(2.5 \times 10^{-4} \mathrm{~mol} / \mathrm{L}\right)$ 的 苂光反应动力学

Figure 2 Fluorescence kinetics of probe $\mathbf{1} \sim \mathbf{5}\left(1.0 \times 10^{-6}\right.$ $\mathrm{mol} / \mathrm{L})$ in reaction with $\mathrm{H}_{2} \mathrm{~S}\left(2.5 \times 10^{-4} \mathrm{~mol} / \mathrm{L}\right)$

(a) Time-dependent emission intensity at $440 \mathrm{~nm}$ of probe 5 after adding $\mathrm{H}_{2} \mathrm{~S}$. (b) The relationship between fluorescence intensity at $440 \mathrm{~nm}$ and reaction time of probe $\mathbf{1} \sim \mathbf{5}$ on treatment with $\mathrm{H}_{2} \mathrm{~S}$ 
表 1 探针 $\mathbf{1} \sim 5$ 与 $\mathrm{H}_{2} \mathrm{~S}$ 的反应动力学参数

Table 1 Kinetic parameters of probe $\mathbf{1} \sim \mathbf{5}$ in reaction with $\mathrm{H}_{2} \mathrm{~S}$

\begin{tabular}{cccc}
\hline Probe & $k_{\text {obs }} / \mathrm{s}^{-1}$ & $K_{2} /\left({\left.\mathrm{L} \cdot \mathrm{mol}^{-1} \cdot \mathrm{s}^{-1}\right)}\right.$ & $T_{1 / 2} / \mathrm{min}$ \\
\hline $\mathbf{1}$ & $3.88 \times 10^{-3}$ & 15.5 & 2.97 \\
$\mathbf{2}$ & $1.74 \times 10^{-3}$ & 6.95 & 6.65 \\
$\mathbf{3}$ & $3.31 \times 10^{-3}$ & 13.2 & 3.49 \\
$\mathbf{4}$ & $1.30 \times 10^{-3}$ & 5.20 & 8.88 \\
$\mathbf{5}$ & $1.94 \times 10^{-3}$ & 7.79 & 5.93 \\
\hline
\end{tabular}

最大发射波长处苂光强度对反应时间作图, 计算并比较 相应的反应速率常数, 结果如图 $2 \mathrm{~b}$ 和表 1 所示, 邻位氟 代叠氮基香豆素 3 与 $\mathrm{H}_{2} \mathrm{~S}$ 的二级反应速率常数 $k_{2}$ 是非氟 代叠氮基香豆素 $\mathbf{2}$ 的 1.9 倍，与探针 $\mathbf{1}$ 相近，表明探针 $\mathbf{1}$ 和 3 与 $\mathrm{H}_{2} \mathrm{~S}$ 的反应速率更匹配. 同时, 氟代双反应探针 5 的二级反应速率常数 $k_{2}$ 是非氟代双反应探针 4 的 1.5 倍, 表明, 引入两个响应速率匹配的反应基团, 可以提 高双反应探针与 $\mathrm{H}_{2} \mathrm{~S}$ 的反应速率.

测试了探针 5 分别与多种具有潜在干扰能力的分子 如金属离子 $\left(\mathrm{Fe}^{3+} 、 \mathrm{Zn}^{2+}\right)$ 、阴离子 $\left(\mathrm{SO}_{4}^{2-} 、 \mathrm{~S}_{2} \mathrm{O}_{3}^{2-}\right.$ 、 $\left.\mathrm{NO}_{2}^{-}\right)$、具有氧化还原能力的活性分子 $\left(\mathrm{H}_{2} \mathrm{O}_{2} 、 \mathrm{HClO}\right.$ 、

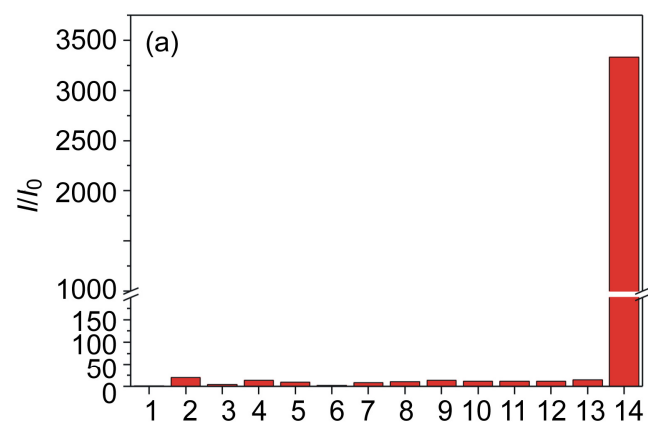

Vc、Cys、Hcy、GSH)等的荧光响应能力，其中除 Cys、 Hcy 浓度为 $1.0 \times 10^{-3} \mathrm{~mol} / \mathrm{L}, \mathrm{GSH}$ 浓度为 $5.0 \times 10^{-3}$ $\mathrm{mol} / \mathrm{L}$ 以外, 其它分子浓度均为 $1.0 \times 10^{-4} \mathrm{~mol} / \mathrm{L}$. 如图 $3 \mathrm{a}$ 所示, 与其它分子相比, 只有 $\mathrm{H}_{2} \mathrm{~S}$ 能够使探针产生显 著的苂光强度变化, 表明双反应探针 5 对 $\mathrm{H}_{2} \mathrm{~S}$ 具有较高 的选择性. 探针的检测灵敏性如图 3b 所示, 随着加入 $\mathrm{H}_{2} \mathrm{~S}$ 浓度增大, 探针溶液荧光显著增强. 以最大苂光强 度对 $\mathrm{H}_{2} \mathrm{~S}$ 浓度作图并拟合, 发现在 $\mathrm{H}_{2} \mathrm{~S}$ 浓度 $0 \sim 1.0 \times$ $10^{-4} \mathrm{~mol} / \mathrm{L}$ 范围内线性关系良好，按照 $3 \sigma / k$ 方法 ${ }^{[12]}$ 计算 探针的检测极限为 $4.0 \times 10^{-8} \mathrm{~mol} / \mathrm{L}$. 上述实验结果表 明, 探针 5 能够对 $\mathrm{H}_{2} \mathrm{~S}$ 高选择性、高灵敏性检测.

\section{2 稳定性}

叠氮还原型探针结构容易被破坏, 在手提式紫外灯 照射下, 对探针 1 5 的稳定性进行了测试. 如图 4a 所 示, 随着紫外照射时间的延长, 邻位氟代叠氮基香豆素 探针 3 很快产生较大强度的苂光, 双反应探针和其它对 照探针分子并未产生明显的苂光变化. 在生理温度甚至 更高的温度 $\left(50{ }^{\circ} \mathrm{C}\right)$, 叠氮还原型探针同样产生一定程

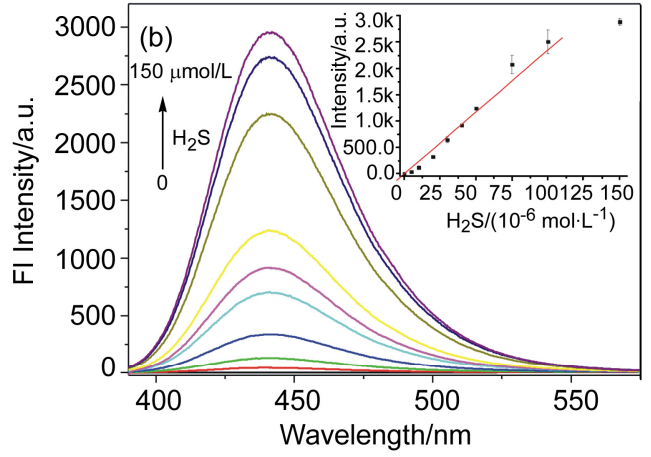

图 3 探针 $\mathbf{5}$ 的选择性和线性关系

Figure 3 Selectivity and linear relationship of probe 5 (a) Fluorescence responses of probe 5 incubated with various analytes. 1, probe $5 ; 2, \mathrm{SO}_{3}^{2-} ; 3, \mathrm{SO}_{4}^{2-} ; 4, \mathrm{~S}_{2} \mathrm{O}_{3}^{2-} ; 5, \mathrm{H}_{2} \mathrm{O}_{2} ; 6, \mathrm{HClO}^{2} ; 7, \mathrm{NO}_{2}^{-} ; 8$, $\mathrm{Zn}^{2+} ; 9, \mathrm{Fe}^{3+} ; 10$, Vc; 11, Cys; 12, Hcy; 13, GSH; 14, $\mathrm{H}_{2} \mathrm{~S}$. (b) Fluorescence response of probe $\mathbf{5}$ with different concentrations of $\mathrm{H}_{2} \mathrm{~S}$ (Insert: The emission intensity at $440 \mathrm{~nm}$ of probe 5 with different concentrations of $\mathrm{H}_{2} \mathrm{~S}$ ). Probe $1.0 \times 10^{-6} \mathrm{~mol} / \mathrm{L}$, Cys and Hcy $1.0 \times 10^{-3} \mathrm{~mol} / \mathrm{L}, \mathrm{GSH} 5 \times 10^{-3}$ $\mathrm{mol} / \mathrm{L}$, other interferents $1.0 \times 10^{-4} \mathrm{~mol} / \mathrm{L} ; \mathrm{H}_{2} \mathrm{~S} 0 \sim 1.50 \times 10^{-4} \mathrm{~mol} / \mathrm{L}$
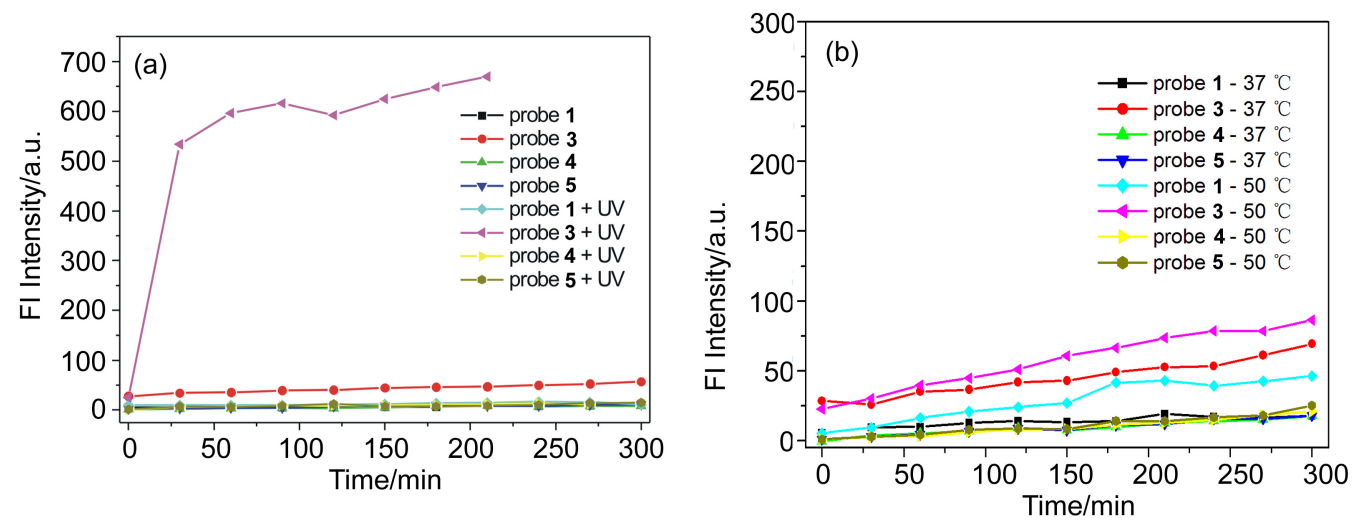

图 4 探针的光稳定 (a) 和热稳定性(b)

Figure 4 Optical stability (a) and thermal stability (b) of the probes

UV lamp power $18 \mathrm{~W}$ for the optical stability test, 37 and $50{ }^{\circ} \mathrm{C}$ for the thermal stability test 
度的荧光增强, 其它探针荧光基本不变. 表明双反应探 针 5 具有良好的高热稳定性.

\section{3 识别机制}

为了研究探针的识别机制, 测试了双反应探针 $\mathbf{5}$ 及 其对照分子与 $\mathrm{H}_{2} \mathrm{~S}$ 反应溶液的紫外可见吸收光谱和高 分辨质谱. 如图 5 所示, 探针 5 结构中叠氮基香豆素和 NBD-哌嗪结构的最大吸收峰分别在 330 和 $500 \mathrm{~nm}$ 处, 随着反应进行, 它们的最大吸收峰分别红移至 360 和 $550 \mathrm{~nm}$, 其变化趋势分别与对照分子 $\mathbf{1}$ 和 $\mathbf{3}$ 的紫外吸收 光谱一致. 探针 5 与 $\mathrm{H}_{2} \mathrm{~S}$ 反应产物的高分辨质谱 $[\mathrm{M}+$ $\mathrm{H}]^{+}$为 306.1248, 与理论值(306.1248)一致. 结合已报道

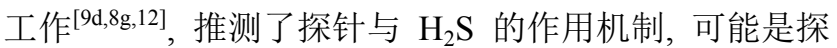
针 5 结构中叠氮基团被 $\mathrm{H}_{2} \mathrm{~S}$ 还原为胺基, NBD-哌嗪与 $\mathrm{H}_{2} \mathrm{~S}$ 发生亲核取代反应(Eq. 2).

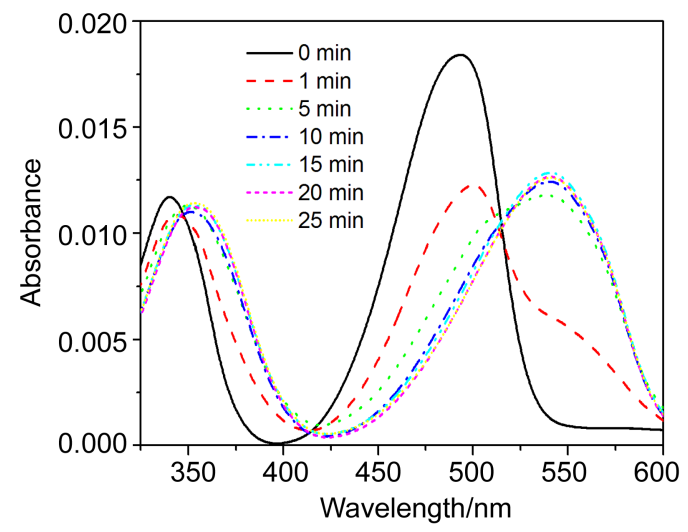

图 5 探针 $5\left(1.0 \times 10^{-6} \mathrm{~mol} / \mathrm{L}\right)$ 与 $\mathrm{H}_{2} \mathrm{~S}\left(1.0 \times 10^{-4} \mathrm{~mol} / \mathrm{L}\right)$ 反应的 紫外吸收光谱

Figure 5 UV-Vis spectrum of probe $5\left(1.0 \times 10^{-6} \mathrm{~mol} / \mathrm{L}\right)$ in reaction with $\mathrm{H}_{2} \mathrm{~S}\left(1.0 \times 10^{-4} \mathrm{~mol} / \mathrm{L}\right)$

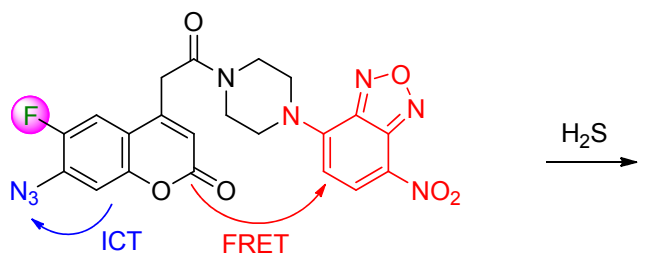<smiles>Nc1cc2oc(=O)cc(CC(=O)N3CCNCC3)c2cc1[O-]</smiles>

\subsection{CBS 酶活性检测}

苂光探针技术已经被用于多种酶的抑制剂高通量 篮选. 文献报道, 真核细胞内 CBS 能够以 $L-C y s$ 为底物 合成内源 $\mathrm{H}_{2} \mathrm{~S}$, 并且羟胺、炔丙基甘氨酸(PPG)和氨基氧 乙酸(AOAA) 可以作为 CBS 的抑制剂调控细胞内源 $\mathrm{H}_{2} \mathrm{~S}$ 的生物合成. 用双反应探针 5 验证了体外表达的人源
CBS ( $\Delta 414-551)$ 的活性和 PPG、AOAA 的抑制能力. 如 图 6 所示, 在加入底物 L-Cys 后，随着孵育时间延长和 CBS 加入量增多, 探针苂光强度逐渐增强(图 6a); 加入 抑制剂 PPG 和 AOAA 后, 探针荧光强度明显降低. 表 明，探针 5 可以用于内源 $\mathrm{H}_{2} \mathrm{~S}$ 合成酶 $\mathrm{CBS}$ 的活性检测和 抑制剂篮选.

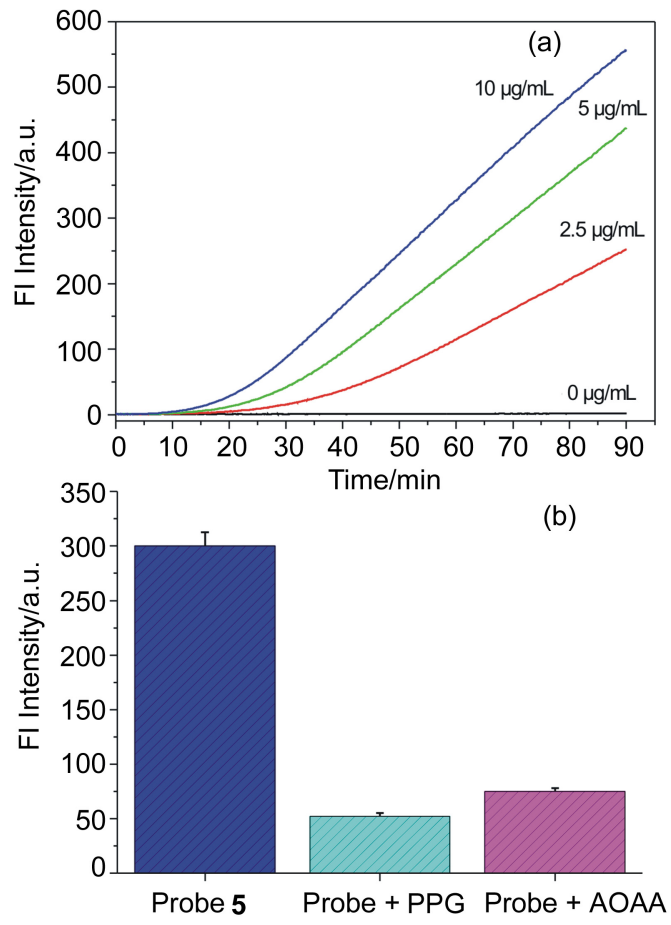

图 6 探针 5 用于 CBS 酶活性检测

Figure 6 Detection of CBS activity using probe 5 (a) The time-dependent emission intensity at $440 \mathrm{~nm}$ of probe $\mathbf{5}, 10$ $\mathrm{mmol} / \mathrm{L} L$-Cys and $0 \sim 10 \mu \mathrm{g}$ CBS enzyme. (b) The fluorescence intensity at $440 \mathrm{~nm}$ of probe 5 before and after treatment with inhibitors. Probe $1.0 \times 10^{-5} \mathrm{~mol} / \mathrm{L}$, CBS $0.01 \mu \mathrm{g} / \mu \mathrm{L}$, inhibitors $1.0 \times 10^{-3} \mathrm{~mol} / \mathrm{L}$

\section{5 细胞成像研究}

探针 5 用于 $\mathrm{HeLa}$ 细胞中 $\mathrm{H}_{2} \mathrm{~S}$ 苂光成像研究. 向细 胞中单独加入探针 $5\left(2.0 \times 10^{-6} \mathrm{~mol} / \mathrm{L}\right)$, 孵育 $30 \mathrm{~min}$, 细 胞产生较弱的苂光(图 $7 \mathrm{a}, 7 \mathrm{~b})$; 探针和 Cys $\left(5.0 \times 10^{-4}\right.$ $\mathrm{mol} / \mathrm{L}$ )共孵育, 细胞荧光增强(图 7c, 7d); 先孵育探针 30 $\min$, 再加入 $\mathrm{H}_{2} \mathrm{~S}\left(2.5 \times 10^{-4} \mathrm{~mol} / \mathrm{L}\right)$ 孵育 $30 \mathrm{~min}$, 细胞荧 光明显增强. 表明, 探针 5 能够用于细胞内 $\mathrm{H}_{2} \mathrm{~S}$ 生物成 像研究.

\section{2 结论}

设计合成了一种响应速率匹配的 FRET-ICT 双反应 $\mathrm{H}_{2} \mathrm{~S}$ 苂光探针 5. 探针可以与 $\mathrm{H}_{2} \mathrm{~S}$ 发生还原和亲核取代 反应，具有良好的选择性，并且探针中两个反应基团的 反应速率相当. 探针可以实现低浓度 $\mathrm{H}_{2} \mathrm{~S}$ 检测，检测极 限为 $40 \mathrm{nmol} / \mathrm{L}$. 此外, 探针能够用于 $\mathrm{CBS}$ 活性检测和 抑制剂篎选, 并且可以检测活细胞中 $\mathrm{H}_{2} \mathrm{~S}$ 生物合成, 具 有潜在的应用价值. 

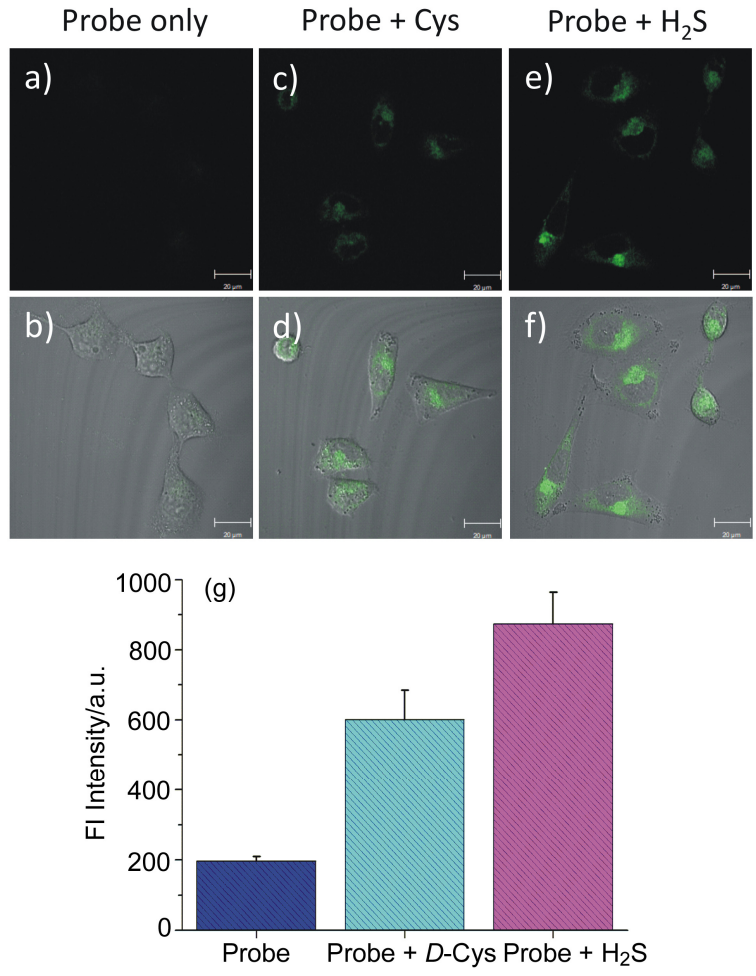

图 7 探针 5 用于 $\mathrm{HeLa}$ 细胞 $\mathrm{H}_{2} \mathrm{~S}$ 苂光成像

Figure 7 Confocal imaging of $\mathrm{H}_{2} \mathrm{~S}$ in HeLa cells using probe 5 (a, b) Cells incubated with probe $\mathbf{5}$ only; (c, d) Cells co-incubated with probe 5 and $D$-cysteine; (e, f) Cells incubated with probe 5 firstly, and then incubated with $\mathrm{H}_{2} \mathrm{~S}$

\section{3 实验部分}

\section{1 仪器与试剂}

Brucker 核磁共振仪(400 MHz 和 $600 \mathrm{MHz}$ ); Varian 7.0 T FTICR-MS 质谱仪(美国 Agilent 公司); Hitachi F-7000 型荧光光谱仪(日本 Hitachi 公司); UV-3600 型分 光光度计(美国 Agilent 公司). 柱层析用硅胶(100 200 目)及 GF254 薄层层析硅胶为青岛海洋化工厂产品; 所 用试剂和溶剂均为国产分析纯或者化学纯试剂, 无水试 剂均按常规方法处理.

\section{2 实验方法}

\subsection{1 探针 5 的合成及表征}

化合物 4 的合成参考文献 $[8 \mathrm{~g}]$ 进行. 将化合物 4 (165 mg, $0.05 \mathrm{mmol}$ )溶解于 $25 \mathrm{~mL}$ 二氯甲烷中, 加入 NBD-Cl (100 mg，0.05 mmol)和三乙胺 $(210 \mu \mathrm{L}, 0.15$ $\mathrm{mmol})$, 室温搅拌过夜. 蒸除溶剂, 柱层析分离 $[V(\mathrm{DCM}) / V(\mathrm{MeOH})=40 / 1]$, 得 $160 \mathrm{mg}$ 淡橘色固体化合 物 5, 产率 65\%. m.p. 193 195 ${ }^{\circ} \mathrm{C} ;{ }^{1} \mathrm{H}$ NMR (400 MHz, DMSO- $\left.d_{6}\right) \delta: 8.55(\mathrm{~d}, J=8.8 \mathrm{~Hz}, 1 \mathrm{H}), 7.74(\mathrm{~d}, J=12.0$ $\mathrm{Hz}, 1 \mathrm{H}), 7.45$ (d, $J=8.0 \mathrm{~Hz}, 1 \mathrm{H}), 6.64$ (d, $J=8.0 \mathrm{~Hz}, 1 \mathrm{H})$, $6.42(\mathrm{~s}, 1 \mathrm{H}), 4.34$ (s, 2H), $4.20(\mathrm{~s}, 2 \mathrm{H}), 4.08$ (s, 2H), 3.92 (s, 2H), $3.78(\mathrm{~s}, 2 \mathrm{H}) ;{ }^{13} \mathrm{C}$ NMR (400 MHz, DMSO- $\left.d_{6}\right) \delta$ : $163.3,159.3,154.8,148.3,145.3,144.8,144.7,144.1$, $136.3,127.5,121.6,223.1,112.2,107.4,103.6,54.9,49.3$, 48.1, 44.9, 40.9. HRMS calcd for $\mathrm{C}_{21} \mathrm{H}_{15} \mathrm{FN}_{8} \mathrm{NaO}_{6}$ 517.0996 , found 517.0995 .

\section{2 .2 光谱测试}

探针 $1 \sim 5$ 用二甲基亚砜(DMSO)配制成 $2.0 \times 10^{-3}$ $\mathrm{mol} / \mathrm{L}$ 的母液, 测试浓度为 $1.0 \times 10^{-6} \mathrm{~mol} / \mathrm{L}$. 选择性测 试所用各种干扰离子均为钠盐或钾盐, 除 Cys 和 Hcy 浓 度为 $1.0 \times 10^{-3} \mathrm{~mol} / \mathrm{L}, \mathrm{GSH}$ 浓度为 $5 \times 10^{-3} \mathrm{~mol} / \mathrm{L}$ 外, 其 它干扰离子测试浓度为 $1.0 \times 10^{-4} \mathrm{~mol} / \mathrm{L}$, 测试溶液为 PBS 缓冲溶液 $\left(1.0 \times 10^{-2} \mathrm{~mol} / \mathrm{L}, \mathrm{pH} 7.4,10 \% \mathrm{CH}_{3} \mathrm{CN}\right)$. 苂光光谱均在 $37{ }^{\circ} \mathrm{C}$ 条件下测试，孵育时间为 $20 \mathrm{~min}$, 样品池为 $1 \mathrm{~cm} \times 1 \mathrm{~cm} \times 4 \mathrm{~cm}$ 石英比色典, 激发波长为 $380 \mathrm{~nm}$, 激发和发射狭缝宽度均为 $5 \mathrm{~nm}$.

\subsubsection{CBS 酶活测试}

人源 $\mathrm{CBS}(\Delta 414-551)$ 活性测试缓冲体系为 Tris- $\mathrm{HCl}$ $(\mathrm{pH} 8.0,0.2 \mathrm{~mol} / \mathrm{L})$, 含有 $5.0 \times 10^{-6} \mathrm{~mol} / \mathrm{L}$ 磷酸吡哆醛 (PLP)、 $1.0 \times 10^{-2} \mathrm{~mol} / \mathrm{L} \mathrm{GSH} 、 0.5 \mathrm{mg} / \mathrm{mL}$ 牛血清蛋白 (BSA). 测试体系总体积 $200 \mu \mathrm{L}$, 其中含有 CBS (0.01 $\mu \mathrm{g} / \mu \mathrm{L}, 2 \mu \mathrm{L})$, 探针 $5\left(1.0 \times 10^{-5} \mathrm{~mol} / \mathrm{L}, 2 \mu \mathrm{L}\right), L$-Cys $\left(1.0 \times 10^{-2} \mathrm{~mol} / \mathrm{L}, 10 \mathrm{mmol} / \mathrm{L}\right)$ 和抑制剂 $\left(1.0 \times 10^{-3} \mathrm{~mol} / \mathrm{L}\right.$, $20 \mu \mathrm{L}) .37{ }^{\circ} \mathrm{C}$ 避光孵育 $1 \mathrm{~h}$.

\section{2 .4 细胞实验}

$\mathrm{HeLa}$ 细胞在高糖培养基中培养，培养基含有体积 分数为 $10 \%$ 的胎牛血清、 $100 \mathrm{U} / \mathrm{mL}$ 盘尼西林、 100 $\mathrm{mg} / \mathrm{mL}$ 链霉素和 $4 \mathrm{mmol} / \mathrm{L} L$-谷氨酰胺. 体积分数为 $5 \%$ $\mathrm{CO}_{2}$, 待生长至融合度为 $75 \%$ 左右时, 加入质量分数为 $0.25 \%$ 的胰酶蛋白消化细胞，进行后续实验.

细胞成像实验: HeLa 细胞接种于成像专用玻底培 养血, 密度为 $2 \times 10^{4} \mathrm{~cm}^{2}$. 探针组仅加入探针 $5(2.0 \times$ $10^{-6} \mathrm{~mol} / \mathrm{L}$ )孵育 $30 \mathrm{~min}$; 内源 $\mathrm{H}_{2} \mathrm{~S}$ 成像组加入探针 5 $\left(2.0 \times 10^{-6} \mathrm{~mol} / \mathrm{L}\right)$ 和底物 $D$-Cys $\left(5.0 \times 10^{-4} \mathrm{~mol} / \mathrm{L}\right)$ 共孵 育 $30 \mathrm{~min}$; 外源 $\mathrm{H}_{2} \mathrm{~S}$ 成像组先加入探针 $5\left(2.0 \times 10^{-6}\right.$ $\mathrm{mol} / \mathrm{L}$ )孵育 $30 \mathrm{~min}, \mathrm{PBS}$ 洗涤三次去除过量探针后, 再 加入 $\mathrm{H}_{2} \mathrm{~S}\left(2.0 \times 10^{-4} \mathrm{~mol} / \mathrm{L}\right)$ 继续孵育 $30 \mathrm{~min}$. 共聚焦苂 光成像激发波长为 $405 \mathrm{~nm}$, 收集范围 450 500 nm.

辅助材料(Supporting Information) 探针 5 的 ${ }^{1} \mathrm{H}$ NMR, ${ }^{13} \mathrm{C} \mathrm{NMR}$ 和 HRMS 图谱，对照分子 $\mathbf{1} \sim \mathbf{4}$ 与 $\mathrm{H}_{2} \mathrm{~S}$ 反应的 紫外吸收光谱。这些材料可以免费从本刊网站 (http://sioc-journal.cn/)上下载.

\section{References}

[1] (a) Szabo, C. Nat. Rev. Drug Discovery 2007, 6, 917. 
(b) Li, L.; Rose, P.; Moore, P. K. Annu. Rev. Pharmacol. Toxicol. 2011, 51, 169 .

(c) Wallace, J. L.; Wang, R. Nat. Rev. Drug Discovery 2015, 14, 329

(d) Kimura, H.; Shibuya, N.; Kimura, Y. Antioxid. Redox Signaling 2012, 17, 45 .

[2] (a) Kabil, O.; Banerjee, R. Antioxid. Redox Signaling 2014, $20,770$.

(b) Kimura, H. Exp. Physiol. 2011, 96, 833.

(c) Yang, G. D.; Wu, L. Y.; Jiang, B.; Yang, W.; Qi, J. S.; Cao, K.; Meng, Q. H.; Asif, K.; Mu, W. T.; Zhang, S. M.; Solomon, H.; Wang, R. Science 2008, 322, 587.

(d) Shibuya, N.; Koike, S.; Tanaka, M.; Ishigami-Yuasa, M.; Kimura, Y.; Ogasawara, Y.; Fukui, K.; Nagahara, N.; Kimura, H. Nat. Commun. 2013, 4, 1366.

[3] (a) Kamoun, P.; Belardinelli, M. C.; Chabli, A.; Lallouchi, K.; Chadefaux-Vekemans, B. Am. J. Med. Genet., Part A 2003, 116, 310 .

(b) Fiorucci, S.; Antonelli, E.; Mencarelli, A.; Orlandi, S.; Renga, B.; Rizzo, G.; Distrutti, E.; Shah, V.; Morelli, A. Hepatology 2005, $42,539$.

[4] (a) Hu, L. F.; Lu, M.; Wu, Z. F.; Wong, P. T.; Bian, J. S. Mol. Pharmacol. 2009, 75, 27.

(b) Li, L.; Bhatia, M.; Moore, P. K. Curr. Opin. Pharmacol. 2006, 6,125 .

[5] (a) Paul, B. D.; Snyder, S. H. Antioxid. Redox Signaling 2015, 22, 411.

(b) Furne, J.; Saeed, A.; Levitt, M. D. Am. J. Physiol.: Regul., Integr. Comp. Physiol. 2008, 295, R1479.

[6] Kolluru, G. K.; Shen, X.; Bir, S. C.; Kevil, C. G. Nitric Oxide 2013, 35,5 .

[7] (a) Gao, M.; Yu, F. B.; Chen. L. X. Prog. Chem. 2014, 26, 1065 (in Chinese).

(高敏, 于法标, 陈令新, 化学进展, 2014, 26, 1065.)

(b) Lin, V. S.; Chen, W.; Xian, M.; Chang, C. J. Chem. Soc. Rev. 2015, 44, 4596

(c) Bruemmer, K. J.; Brewer, T. F.; Chang, C. J. Curr. Opin. Chem. Biol. 2017, 39, 17 .

(d) Chen, Y.; Wei, T.; Zhang, Z.; Zhang, W.; Lü, J. Chin. Chem. Lett. 2017, 28, 1957.

[8] (a) Lippert, A. R.; New, E. J.; Chang, C. J. J. Am. Chem. Soc. 2011, 133, 10078

(b) Peng, H. J.; Cheng, F. Y.; Dai, C. F.; Adrienne L. K.; Benjamin, L. P.; David, J. L.; Wang, B. H. Angew. Chem., Int. Ed. 2011, 50, 9672 .

(c) Chen, S.; Chen, Z. J.; Ren, W.; Ai, H. W. J. Am. Chem. Soc. 2012, 134, 9589.

(d) Montoya, L. A.; Pluth, M. D. Chem. Commun. 2012, 48, 4767.

(e) Sun, W.; Fan, J. L.; Hu, C.; Cao, J. F.; Zhang, H.; Xiong, X. Q.; Wang, J. Y.; Cui, S.; Sun, S. G.; Peng, X. J. Chem. Commun. 2013, 49, 3890.

(f) Zhang, J. Y.; Guo, W. Chem. Commun. 2014, 50, 4214. (g) Wei, C.; Wang, R. Y.; Wei, L.; Cheng, L. H.; Li, Z. F.; Xi, Z.; Yi, L. Chem.-Asian J. 2014, 9, 3586.

(h) Zhu, Z. T.; Li, Y. Y.; Wei, C.; Wen, X.; Xi, Z.; Yi, L. Chem.Asian J. 2016, 11, 68.

(i) He, P.; Tang, L. J.; Zhong, K. L.; Hou, S. H.; Yan, X. M. Chin. J. Org. Chem. 2017, 37, 423 (in Chinese).

(何平, 汤立军, 钟克利, 侯淑华, 燕小梅, 有机化学, 2017, 37, 423.)

[9] (a) Liu, C. R.; Pan, J.; Li, S.; Zhao, Y.; Wu, L. Y.; Berkman, C. E.; Whorton, A. R.; Xian, M. Angew. Chem., Int. Ed. 2011, 50, 10327.

(b) Cao, X. W.; Lin, W. Y.; Zheng, K. B.; He, L. W. Chem. Commun. 2012, 48, 10529

(c) Huang, Z. J.; Ding, S. S.; Yu, D. H.; Huang, F. H.; Feng, G. D. Chem. Commun. 2014, 50, 9185.

(d) Wei, C.; Wei, L.; Xi, Z.; Yi, L. Tetrahedron Lett. 2013, 54, 6937. (e) Wei, C.; Zhu, Q.; Liu, W. W.; Chen, W. B.; Xi, Z.; Yi, L. Org. Biomol. Chem. 2014, 12, 479.

(f) Zhao, C. C.; Zhang, X. L.; Li, K. B.; Zhu, S. J.; Guo, Z. Q.; Zhang, L. L.; Wang, F. Y.; Fei, Q.; Luo, S. H.; Shi, P.; Tian, H.; Zhu, W. H. J. Am. Chem. Soc. 2015, 137, 8490.

(g) Zhou, C.; Qiu, B.; Zeng, Y.; Chen, J. P.; Yu, T. J.; Li, Y. Chin. J. Org. Chem. 2017, 37, 92 (in Chinese).

(周婵，邱波，曾毅，陈金平，于天君，李嫕，有机化学, 2017, 37, 92.)

[10] (a) Qian, Y.; Karpus, J.; Kabil, O.; Zhang, S. Y.; Zhu, H. L.; Banerjee, R.; Zhao, J.; He, C. Nat. Commun. 2011, 2, 495.

(b) Li, X.; Zhang, S.; Cao, J.; Xie, N.; Liu, T.; Yang, B.; He, Q. J.; Hu, Y. Z. Chem. Commun. 2013, 49, 8656.

(c) Liu, J.; Sun, Y. Q.; Zhang, J. Y.; Yang, T.; Cao, J. B.; Zhang, L. S.; Guo, W. Chem.-Eur. J. 2013, 19, 4717.

(d) Chen, Y. C.; Zhu, C. C.; Yang, Z. H.; Chen, J. J.; He, Y. F.; Jiao, Y.; He, W. J.; Qiu, L.; Cen, J. J.; Guo, Z. J. Angew. Chem., Int. Ed. 2013, 52, 1688.

[11] (a) Sasakura, K.; Hanaoka, K.; Shibuya, N.;Mikami, Y.; Kimura, Y.; Komatsu, T.; Ueno, T.; Terai, T.; Kimura, H.; Nagano, T. J. Am. Chem. Soc. 2011, 133, 18003.

(b) Qu, X. Y.; Li, C. J.; Chen, H. C.; Mack, J.; Guo, Z. J.; Shen, Z. Chem. Commun. 2013, 49, 7510.

(c) Zhong, K. L.; Zhao, J.; Li, Q. Y.; Hou, S. H.; Tang, Y. W.; Bian, Y. J.; Tang, L. J. Chin. J. Org. Chem. 2018, 38, 1786 (in Chinese). (钟克利, 赵杰, 李秋荣, 侯淑华, 汤轶伟, 边延江, 汤立军, 有 机化学, 2018, 38, 1786.)

[12] (a) Zhang, C. Y.; Wei, L.; Wei, C.; Zhang, J.; Wang, R. Y.; Xi, Z.; Yi , L. Chem. Commun. 2015, 51, 7505.

(b) Wei, C.; Wang, R. Y.; Zhang, C. Y.; Xu, G. C.; Li, Y. Y.; Zhang, Q. Z.; Li, L. Y.; Yi, L.; Xi, Z. Chem.-Asian J. 2016, 11, 1376.

(c) Ma, C.; Wei, C.; Li, X. Y.; Zheng, X. Y.; Chen, B.; Wang, M.; Zhang, P. Z.; Li, X. L. Dyes Pigm. 2019, 162, 624.

(d) Montoya, L. A.; Pearce, T. F.; Hansen, R. J.; Zakharov, L. N.; Pluth, M. D. J. Org. Chem., 2013, 78, 6550. 